\section{Acurácia de informações sobre classes de medicamentos obtidas com questionário postal aplicado a idosos - Rio de Janeiro, RJ}

\section{Accuracy of drug class information obtained from a postal questionnaire to elderly respondents}

Maria Estrella L. Vasconcelos'

Maria J. M. Fonsecal"

Suely Rozenfeld"

Francisco A. Acurcio"'

' PAM 404 Brigadeiro Lima e Silva. Rio de Janeiro, RJ

"Escola de Saúde Pública Sérgio Arouca, Fundação Oswaldo Cruz, Rio de Janeiro, RJ

"'Faculdade de Farmácia da Universidade Federal de Minas Gerais. Belo Horizonte, MG

Artigo baseado em dissertação de mestrado de Maria Estrella López Vasconcelos, Confiabilidade e validade das informaçōes sobre doenças e medicamentos em aposentados pelo INSS, 2007, Escola Nacional de Saúde Pública Sérgio Arouca, Fundação Oswaldo Cruz, Rio de Janeiro, RJ, Brasil.

Correspondência: Maria Estrella L. Vasconcelos. PAM 404 Brigadeiro Lima e Silva. Rua Professor José de Souza Herdy, 389 - Jardim 25 de Agosto, Duque de Caxias, RJ. E-mail: estrellalopez@cremerj.org.br

\section{Resumo}

Objetivos: verificar a confiabilidade e a validade das informações sobre medicamentos obtidas em questionário postal, respondido por idosos, sendo a entrevista face a face o padrão-ouro. Métodos: estudo seccional (Perfil de Utilização de Medicamentos por Aposentados Brasileiros), onde foram utilizadas duas abordagens (postal e domiciliar) para coleta de informações de aposentados pelo Instituto Nacional do Seguro Social (INSS) com sessenta anos de idade ou mais. Foram utilizadas também as estatísticas kappa (simples (k), ajustado (PABAK) e ponderado), índices de correlação intra-classe, indicadores de sensibilidade e especificidade, e o gráfico de Luiz et al. Resultados: 234 idosos ( $M=42 \%$; $F=58 \%$ ) responderam às duas abordagens (média $=71,7$ anos). A concordância entre postal $\mathrm{e}$ entrevista domiciliar foi excelente $(\mathrm{k}=0,94)$ para hipoglicemiantes; muito boa $(\mathrm{k}=0,83$ 0,82 ) para inibidores da enzima conversora de angiotensina e anti-hipertensivos; boa $(\mathrm{k}=0,71)$ para diuréticos; e razoável $(\mathrm{k}=$ 0,47) para antiinflamatórios não esteróides. A concordância foi boa $(k=0,61)$ para o número total de medicamentos usados. A validade da abordagem postal foi elevada, às vezes total, para os fármacos empregados no tratamento do diabetes (sensibilidade $\mathrm{e}$ especificidade $=100 \%$ ), seguidos dos antihipertensivos. Os menores valores obtidos foram para antiinflamatórios não esteróides (sensibilidade $=64 \%$; especificidade $=88 \%$ ). Conclusão: a abordagem postal pode ser usada para se obter informações acuradas sobre classes de medicamentos usados por população com idade igual ou superior a 60 anos, considerando idosos com perfil social semelhante ao dos beneficiários do INSS.

Palavras-chave: Confiabilidade. Validade. Estudos de concordância. Utilização de medicamentos. Serviços de saúde para idosos. Aposentados. Questionário postal. Entrevista face a face. Inquérito postal. Estudos seccionais. 


\section{Abstract}

Objectives: to determine the reproducibility and validity of information on medication obtained in a postal questionnaire, with face-to-face interviews providing the gold standard. Methods: cross-sectional study (Perfil de Utilização de Medicamentos por Aposentados Brasileiros), using two approaches (postal and home visit) to collect information from retirees who were 60 years old or older and beneficiaries of Brazil's national social security system (INSS). Statistics used were: simple kappa $(\mathrm{k})$, adjusted kappa (PABAK), weighted kappa, intraclass correlation coefficients, indicators of sensitivity and specificity, and Luiz et al's graph method. Results: 234 elderly (male $=42 \%$; female $=58 \%$; mean $=71.7$ years) answered both approaches. Concordance between the postal and face-to-face interview approaches was excellent $(\mathrm{k}=0.94)$ for hypoglycemics; very good ( $k=0.83-0.82)$ for angiotensin-converting enzyme inhibitors and anti-hypertensives; good $(\mathrm{k}=0.71)$ for diuretics; and reasonable $(\mathrm{k}=0.47)$ for nonsteroidal anti-inflammatories. Concordance was good $(k=0.61)$ for information on the total number of medications used. The validity of the postal approach was high, at times total, for the drugs used in treating diabetes (sensitivity and specificity $=100 \%$ ), followed by anti-hypertensives. The lowest values obtained were for non-steroidal anti-inflammatories (sensitivity $=64 \%$; specificity $=88 \%$ ). Conclusion: the postal approach can be used to obtain accurate information on classes of medications used by a population aged 60 years or more with a social profile similar to that of social security beneficiaries.

Keywords: Reproducibility. Validity. Concordance studies. Drug use. Health service for the elderly. Medications. Postal questionnaire. Face-to-face interview. Postal survey. Cross-sectional studies.

\section{Introdução}

A realização de estudos seccionais para avaliar medicamentos usados por uma população é tarefa desafiadora, em razão do custo, do viés de memória, das dificuldades inerentes à coleta de dados válidos e confiáveis sobre posologia, duração do tratamento, modo de utilização, multiplicidade de nomes comerciais para a mesma formulação e dos problemas para classificar e analisar os produtos consumidos. Quando os participantes desses estudos são idosos, pode haver dificuldade para lembrar os nomes dos medicamentos que estão utilizando, o que exige cuidado redobrado na obtenção de informações ${ }^{1}$.

As entrevistas face a face têm sido consideradas ao longo do tempo como a abordagem de escolha para a coleta de dados em estudos epidemiológicos ${ }^{2}$, porém são dispendiosas e apresentam um grau maior de dificuldade na sua realização quando comparadas às entrevistas por telefone $\mathrm{e}$ aos questionários postais. Além disso, a sensação de insegurança gerada pela violência urbana nos grandes centros dificulta a realização das entrevistas domiciliare ${ }^{3} \mathrm{e}$ desencoraja a participação individual dos idosos nesses estudos ${ }^{4}$.

O questionário postal representa um método alternativo econômico e de mais fácil realização, quando comparado às entrevistas face a face e às realizadas por telefone $^{5}$. Por outro lado, algumas limitações na abordagem postal são apontadas, tais como: impossibilidade de garantir que as respostas tenham sido dadas pelo indivíduo selecionado $^{6}$ e taxas de resposta menores do que as obtidas com entrevistas face a face ${ }^{7}$, em virtude dos baixos níveis de escolaridade. Pessoas com nível socioeconômico mais baixo podem ser sub-representadas em entrevistas postais. Além disso, os participantes podem responder de modo diferente as mesmas questões, por estarem, ou não, na presença de entrevistadores ${ }^{7}$. Os entrevistados podem se sentir mais à vontade em responder a questões embaraçosas num questionário, ou serem mais motivados 
a responder determinadas questões pelo estímulo dos entrevistadores.

O uso do questionário postal não é recente. Barber e colaboradores, em 1980, utilizaram esta técnica para identificar idosos na comunidade com necessidades de cuidados de saúde ${ }^{8}$.Vários estudos com idosos, realizados com diferentes propósitos, apresentaram taxas de resposta e medidas de confiabilidade e validade satisfatórias (Tabela 1).

Não foi possível identificar, no Brasil, estudos sobre o emprego de questionários postais para a coleta de dados sobre o uso de medicamentos por idosos.

Aqui pretende-se verificar a confiabilidade e a validade das informações sobre medicamentos, obtidas em questionários postais, em uma população de aposentados pelo sistema oficial de previdência social residentes na cidade do Rio de Janeiro, comparadas às obtidas em entrevistas face a face.

\section{Métodos}

\section{O inquérito}

Os dados analisados provêm de um inquérito denominado Perfil de Utilização de Medicamentos por Aposentados Brasileiros. Trata-se de um estudo que utilizou dois tipos de abordagem: questionário postal para a coleta de informações em três amostras, no nível nacional, no município de Belo Horizonte e no município do Rio de Janeiro, e entrevista domiciliar em amostras dos municípios citados.

A população alvo do estudo era composta por indivíduos aposentados com idade igual ou superior a sessenta anos, pertencentes ao Regime Geral de Previdência Social do INSS (Instituto Nacional do Seguro Social). As amostras foram aleatórias simples, sem reposição, extraídas do cadastro nacional da DATAPREV, Empresa de Tecnologia e Informações da Previdência Social, em dezembro de 2002.

Neste artigo, é analisada a amostra referente ao município do Rio de Janeiro, onde foram sorteados 800 indivíduos para o recebimento do questionário postal e para o agendamento de entrevista no domicílio.

Os questionários postal e domiciliar eram compostos por três blocos de perguntas sobre características sociodemográficas dos participantes, indicadores das condições de saúde e de uso de serviços de saúde, e uso de medicamentos. É importante destacar que, no questionário postal, constava a orientação para o respondente colocar "na sua frente as embalagens, ou as receitas, de todos os remédios" que usou nos últimos 15 dias. Na entrevista face a face o entrevistador pedia aos participantes que trouxessem todos os medicamentos, embalagens, bulas e receitas do que estavam utilizando nos últimos 15 dias e registrava as informações.

Os questionários postais foram enviados duas vezes, nos dias 17 de janeiro e 24 de fevereiro de 2003, com porte pago para retorno, e foram aceitos os que chegaram até o dia anterior à entrevista domiciliar, iniciada em 19 de março e concluída em agosto de 2003.

O controle de qualidade da coleta de informações foi realizado pela replicação das amostras domiciliares em subamostras de até $10 \%$ das entrevistas feitas por cada entrevistador, e pelo controle estatístico de algumas informações, que consistiu na confrontação de alguns dados coletados nessas entrevistas com os dos registros da DATAPREV.

Os medicamentos foram classificados com o auxílio do Dicionário de Especialidades Farmacêuticas (DEF) e do Anatomical Therapeutic Chemical Classification System (ATC).

A cobertura do inquérito domiciliar foi de $65,5 \%$, e a taxa de resposta ao postal foi de $34,4 \%$.

A comparação entre respondentes e não respondentes ao inquérito postal sugere não haver diferenças significativas, tendo em vista os $p$-valores (p) calculados para as variáveis: sexo e idade $(\mathrm{p}=0,65)$, local de moradia $(\mathrm{p}=0,53)$, e valor do benefício recebido do INSS $(\mathrm{p}=0,14)^{9}$. As perdas inevitáveis das entrevistas domiciliares $(9,4 \%)$ foram devi- 
Tabela 1 - Comparação dos resultados entre abordagem postal e entrevista face a face, em estudos de saúde com idosos.

Table 1 - Comparison of results from postal approach and face-to-face interviews, in health studies with elderly people.

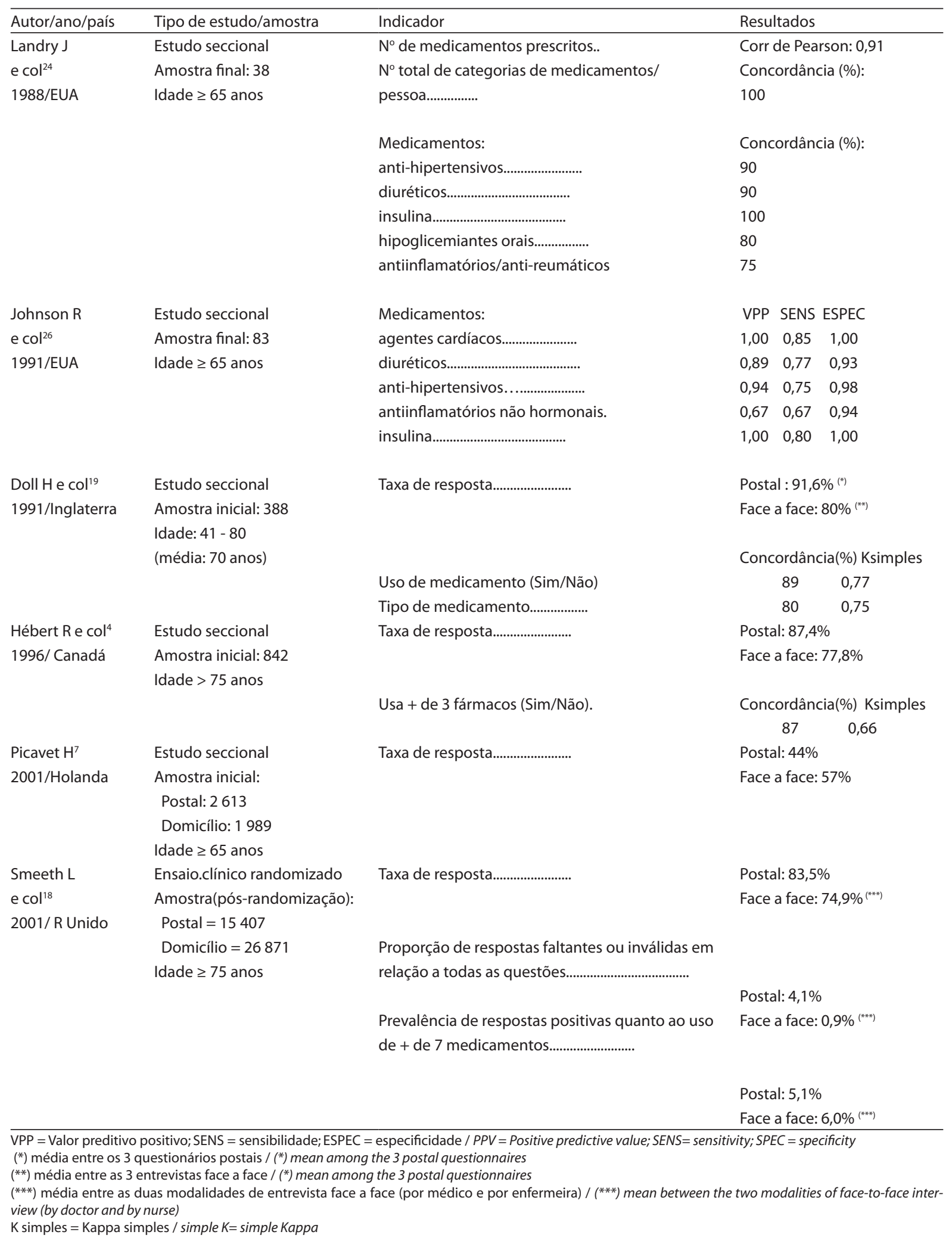


das a óbitos (22 pessoas), mudanças para outros municípios (51 pessoas) e sorteios indevidos (2 pessoas). Dentre as perdas evitáveis das entrevistas domiciliares (25\%), as mais importantes foram as recusas (77) e a não-localização do indivíduo em virtude de mudança para endereço desconhecido (65). Não houve diferença significativa $(p=0,20)$ entre o percentual de perdas inevitáveis das entrevistas domiciliares no grupo dos que responderam apenas ao postal (37\%) e no grupo dos que não responderam a nenhuma abordagem (26\%).

Informações detalhadas sobre a metodologia podem ser encontradas em Acurcio e colaboradores ${ }^{9}$.

O projeto foi aprovado pelos Comitês de Ética em Pesquisa da Fundação Oswaldo Cruz e da Universidade Federal de Minas Gerais.

\section{Análise de dados}

Para fins de análise no presente estudo, foram considerados apenas os idosos que responderam às duas abordagens $(\mathrm{n}=234)$.

As variáveis estudadas foram: idade (6069, 70-79, 80 anos ou mais), sexo, escolaridade (nunca estudou, $1^{0}$ grau incompleto, $1^{0}$ grau completo, $2^{0}$ grau completo, superior), e medicamentos utilizados nos últimos 15 dias (todos os medicamentos que estavam em uso ou que foram utilizados nesse período).

A partir da variável medicamentos utilizados nos últimos 15 dias foi criada a variável número de medicamentos, que foi estratificada em três categorias (nenhum, 1 a 4, 5 ou mais).

Para examinar a validade das informações sobre medicamentos foram selecionados os anti-hipertensivos, os diuréticos, os beta-bloqueadores adrenérgicos, os bloqueadores seletivos do canal de cálcio, os inibidores da enzima conversora de angiotensina, os hipolipemiantes, os hipoglicemiantes orais, as insulinas e os antiinflamatórios não esteróides.

Com o objetivo de descrever a amostra, foi testada a hipótese de homogeneidade entre as proporções das respostas nas duas abordagens, para cada variável, utilizandose o teste qui-quadrado de Pearson $\left({ }^{2}\right)$ e considerando um nível de significância de $5 \%^{10}$.

Para a análise de concordância das variáveis idade, escolaridade, e número de medicamentos utilizados, sendo elas ordinais com mais de duas categorias, utilizou-se, além da estatística kappa simples $^{11}$ (k), o índice kappa ponderado com pesos calculados em função do quadrado da distância entre a diagonal principal e as respectivas categorias ${ }^{12}$. Para a análise da concordância das informações referentes às classes dos medicamentos selecionados foram utilizados o percentual de concordância simples, o índice kappa simples, e o kappa ajustado pelo efeito de prevalências e viés do observador ${ }^{11}$ (PABAK). Os resultados foram classificados segundo Byrt $\mathrm{T}^{13}$ : a concordância é pobre quando o índice estiver entre 0,00 e 0,20 , leve entre 0,21 e 0,40 , razoável entre 0,41 e 0,60 , boa entre 0,61 e 0,80 , muito boa entre 0,81 e 0,92 , e excelente entre 0,93 e 1,00.

Para a análise da concordância da informação sobre número de medicamentos, foram utilizados ainda o coeficiente de correlação intra-classe (CCIC), obtido pelo modelo "two-way" de análise de variância (ANOVA), assumindo serem os observadores fixos - modelo "two-way" misto ${ }^{14}$ (a unidade de análise para o cálculo desse coeficiente de correlação foi a média por indivíduo). Também foi utilizado o método desenvolvido por Luiz e colaboradores ${ }^{15,16}$,que utiliza a análise de sobrevida de Kaplan-Meier, na qual as diferenças no número de medicamentos entre as duas abordagens aparecem em valores absolutos (limites de concordância) no eixo horizontal, e como proporções de discordâncias no eixo vertical. O gráfico permite identificar limites dentro dos quais se aceita certa porcentagem de discordância na amostra.

Para verificar a validade das informações contidas no questionário postal sobre as classes de medicamentos selecionados foram utilizados os indicadores de sensibi- 
lidade e especificidade e o valor preditivo positivo (VPP) ${ }^{17}$, tendo como padrão-ouro a entrevista face a face.

A análise estatística foi feita com o programa $\mathrm{R}$, versão 2.3.1.

\section{Resultados}

Na Tabela 2 são apresentadas as características gerais dos participantes, segundo a resposta obtida em cada abordagem. Entre as variáveis estudadas, não houve diferenças significativas entre as proporções encontradas nas abordagens postal e domiciliar, embora na entrevista os indivíduos tenham referido escolaridade mais elevada e uso de medicamentos menos intenso.

Dos 234 idosos, 98 (42\%) eram homens e 136 (58\%) eram mulheres. A média de idade foi de 71,8 anos entre os homens e de 71,5 entre as mulheres. Mais da metade da amostra não completou o primeiro grau, $63,5 \%$ segundo o postal e $56,0 \%$ segundo a entrevista $(p=0,15)$; e apenas uma pequena parte dos respondentes completou o ensino superior, $9,1 \%$ segundo o postal e $10 \%$ segundo a entrevista. Houve variação entre as abordagens na menção quanto ao número de medicamentos utilizados pelos participantes, embora sem significância estatística $(\mathrm{p}=0,10)$. O percentual de sujeitos que não usaram nenhum medicamento foi maior na entrevista (13,2\%) do que no postal ( $8,5 \%)$, e o percentual dos que usaram cinco ou mais produtos foi maior no postal $(48,7 \%)$ do que na entrevista $(40,2 \%)$.

Na Tabela 3 estão apresentados os resultados da análise de confiabilidade das

Tabela 2 - Características gerais dos aposentados pelo Instituto Nacional do Seguro Social, com 60 anos de idade ou mais, segundo a abordagem, Rio de Janeiro, RJ, Brasil, 2003.

Table 2 - Overall characteristics of social security retirees at least 60 years old, by approach, Rio de Janeiro City, Brazil, 2003.

\begin{tabular}{|c|c|c|c|c|c|}
\hline \multirow[t]{2}{*}{ Variáveis } & \multicolumn{2}{|c|}{ Postal } & \multicolumn{2}{|c|}{ Entrevista } & \multirow[t]{2}{*}{$p$-valor (*) } \\
\hline & $N$ & $\%$ & $\boldsymbol{N}$ & $\%$ & \\
\hline \multicolumn{6}{|l|}{ Sexo } \\
\hline Homens & 98 & 41,9 & 98 & 41,9 & 0,925 \\
\hline Mulheres & 136 & 58,1 & 136 & 58,1 & \\
\hline \multicolumn{6}{|l|}{ Grupo etário } \\
\hline $60-69$ anos & 103 & 45,4 & 105 & 44,9 & \\
\hline $70-79$ anos & 88 & 38,8 & 91 & 38,8 & 0,991 \\
\hline 80 anos ou + & 36 & 15,9 & 38 & 16,3 & \\
\hline \multicolumn{6}{|l|}{ Escolaridade } \\
\hline Nunca estudou & 29 & 12,6 & 27 & 11,7 & \\
\hline $1^{\circ}$ grau incompleto & 117 & 50,9 & 105 & 44,3 & \\
\hline $1^{\circ}$ grau completo & 35 & 14,8 & 37 & 16,1 & 0,572 \\
\hline $2^{\circ}$ grau completo & 29 & 12,6 & 41 & 17,8 & \\
\hline Superior & 21 & 9,1 & 23 & 10,0 & \\
\hline \multicolumn{6}{|c|}{$\begin{array}{l}\text { Número de medicamentos } \\
\text { utilizados nos últimos } 15 \\
\text { dias }\end{array}$} \\
\hline \multicolumn{6}{|l|}{ Nenhum } \\
\hline 1 a 4 & 20 & 8,5 & 31 & 13,2 & 0,096 \\
\hline \multirow[t]{2}{*}{5 ou mais } & 100 & 42,7 & 109 & 46,6 & \\
\hline & 114 & 48,7 & 94 & 40,2 & \\
\hline
\end{tabular}

$(*)$ teste qui-quadrado de Pearson

$(*)$ Pearson's chi-square test 
variáveis idade, escolaridade e número de medicamentos utilizados. A informação sobre a idade foi a que apresentou a maior concordância (kappa ponderado = 0,97 - IC 95\%: 0,92-1,00). A concordância entre as informações obtidas nas duas abordagens sobre o número de medicamentos nos últimos 15 dias foi boa, embora com intervalo de confiança e diferenças maiores entre o percentual de concordância simples $(70,5 \%)$ e o kappa ponderado $(0,61$ - IC 95\%: 0,51-0,70). O coeficiente de correlação intra-classe para a variável número de medicamentos foi 0,63 (IC 95\%: 0,52-0,71).

A concordância/discordância sobre o número de medicamentos foi também analisada por meio de análise de sobrevida. Na Figura 1 observa-se que em $80 \%$ da amostra a discordância da informação sobre o número de medicamentos entre os dois métodos é de até três medicamentos.
A figura permite constatar que em metade da amostra não há divergência entre as duas abordagens ou há entre elas diferença de um único medicamento mencionado.

$\mathrm{Na}$ Tabela 4 estão apresentados os resultados da análise de confiabilidade para as classes de medicamentos. Os hipoglicemiantes orais foram os que apresentaram maior concordância (percentual de concordância $=98,7 \%$ e kappa simples $(\mathrm{k})=0,94)$, juntamente com as insulinas (percentual de concordância $=99,6 \%$ e k = $0,86)$, os inibidores da enzima conversora de angiotensina (percentual de concordância $=92,3 \%$ e $\mathrm{k}=0,83$ ), e os anti-hipertensivos (percentual de concordância $=98,3 \%$ e k = 0,82 ). Os diuréticos apresentaram o menor valor de $\mathrm{k}(0,71)$ dentre os medicamentos do sistema cardiovascular, e a classe dos antiinflamatórios não esteróides foi a que obteve a menor concordância $(k=0,47)$.

Tabela 3 - Comparação da informação sobre a idade, escolaridade e número de medicamentos utilizados nos últimos quinze dias, segundo a abordagem, por aposentados pelo Instituto Nacional do Seguro Social, com 60 anos de idade ou mais, Rio de Janeiro, RJ, Brasil, 2003.

Table 3 - Comparison of information on age, schooling and number of drugs used in the previous fifteen days, by approach, among social security retirees at least 60 years old, Rio de Janeiro City, Brazil, 2003.

\begin{tabular}{|c|c|c|c|c|c|c|c|c|}
\hline \multirow{2}{*}{$\begin{array}{l}\text { Postal } \\
\text { Idade }\end{array}$} & \multicolumn{5}{|c|}{ Entrevista } & \multirow{2}{*}{$\begin{array}{c}\text { concordância } \\
\text { (\%) }\end{array}$} & \multirow{2}{*}{$\begin{array}{c}\text { Kappa } \\
\text { ponderado }\end{array}$} & \multirow{2}{*}{$\begin{array}{c}\text { IC } \\
(95 \%) \\
\end{array}$} \\
\hline & $60-69$ & \multicolumn{2}{|c|}{$70-79$} & \multicolumn{2}{|l|}{80 e mais } & & & \\
\hline 60-69 anos & 100 & \multicolumn{2}{|l|}{2} & 1 & & & & \\
\hline 70-79 anos & 2 & \multicolumn{2}{|l|}{86} & 0 & & 97,8 & 0,97 & $0,92-1,00$ \\
\hline 80 anos e mais & 0 & \multicolumn{2}{|l|}{0} & 36 & & & & \\
\hline Total & 102 & \multicolumn{2}{|l|}{88} & 37 & & & & \\
\hline Escolaridade & $\begin{array}{l}\text { Nunca } \\
\text { estudou }\end{array}$ & $\begin{array}{l}1^{0} \mathrm{grau} \\
\text { incom }\end{array}$ & $\begin{array}{l}1^{0} \mathrm{grau} \\
\text { compl }\end{array}$ & $\begin{array}{l}2^{0} \mathrm{grau} \\
\text { compl }\end{array}$ & Superior & & & \\
\hline Nunca estudou & 21 & 7 & 1 & 0 & 0 & & & \\
\hline $1^{\circ} \mathrm{grau}$ incompleto & 6 & 90 & 17 & 3 & 1 & & & \\
\hline $1^{0}$ grau completo & 0 & 4 & 19 & 11 & 0 & 75,7 & 0,87 & $0,82-0,92$ \\
\hline $2^{0}$ grau completo & 0 & 1 & 0 & 25 & 3 & & & \\
\hline Superior & 0 & 0 & 0 & 2 & 19 & & & \\
\hline $\begin{array}{l}N^{\circ} \text { de medicamentos } \\
\text { utilizados nos últimos } \\
15 \text { dias }\end{array}$ & Nenhum & $1 \mathrm{a}$ & 4 & 5 ou mais & & & & \\
\hline Nenhum & 15 & 5 & & 0 & & & & \\
\hline 1 a 4 & 12 & 72 & 2 & 16 & & 70,5 & 0,61 & $0,51-0,70$ \\
\hline 5 ou mais & 4 & 32 & & 78 & & & & \\
\hline
\end{tabular}

$\mathrm{IC}=$ Intervalo de Confiança $/ \mathrm{Cl}=$ Confidence Interval 


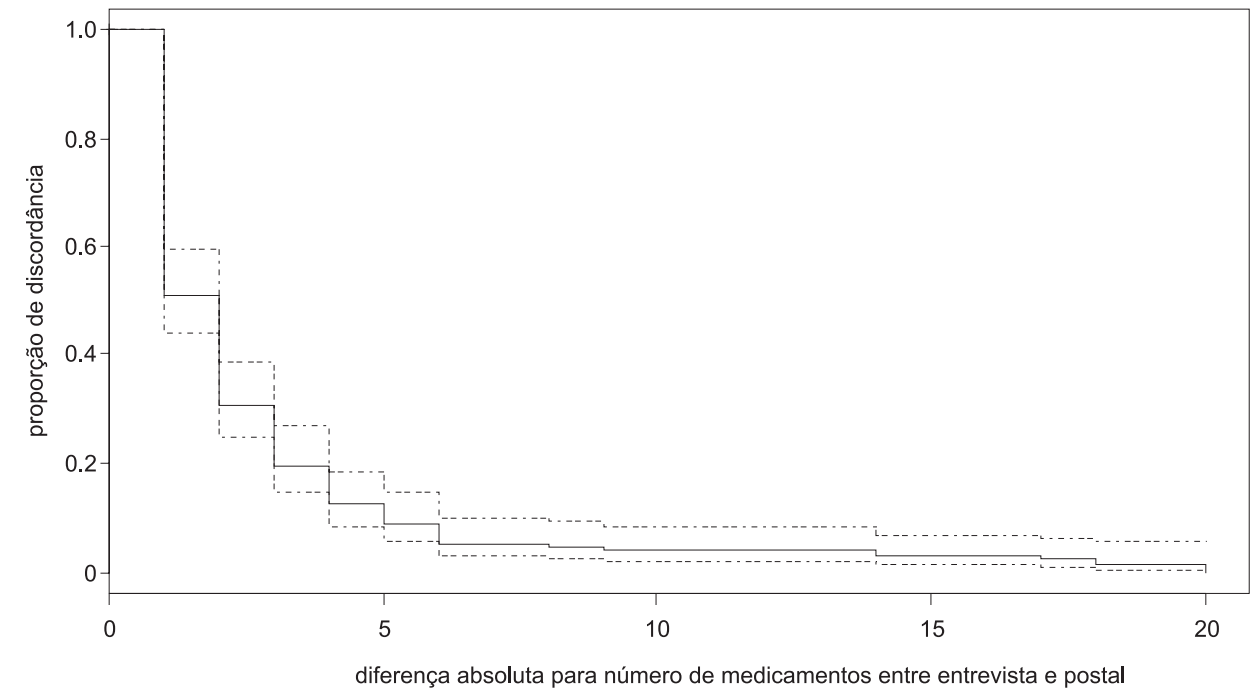

Figura 1 - Proporção de discordância para número de medicamentos entre entrevista e postal, por aposentados pelo Instituto Nacional do Seguro Social, com 60 anos de idade ou mais, Rio de Janeiro, RJ, Brasil, 2003.

Figure 1 - Proportion of disagreement in the number of drugs, between live interview and postal questionnaire, by social security retirees at least 60 years old, Rio de Janeiro City, Brazil, 2003.

Tabela 4 - Comparação da informação sobre o uso de classes de medicamentos, segundo a abordagem, por aposentados pelo Instituto Nacional do Seguro Social, com 60 anos de idade ou mais, Rio de Janeiro, RJ, Brasil, 2003. Table 4 - Comparison of information on drug class use, by approach, among social security retirees at least 60 years old, Rio de Janeiro City, Brazil, 2003.

\begin{tabular}{|c|c|c|c|c|c|c|c|}
\hline \multirow{2}{*}{ Medicamentos } & \multirow{2}{*}{ Postal } & \multicolumn{2}{|c|}{ Entrevista } & \multirow{2}{*}{$\begin{array}{c}\% \text { de } \\
\text { concordância }\end{array}$} & \multirow{2}{*}{$\begin{array}{l}\text { Kappa } \\
\text { simples }\end{array}$} & \multirow{2}{*}{$\begin{array}{c}\text { IC } \\
(95 \%)\end{array}$} & \multirow{2}{*}{$\begin{array}{c}\text { PABAK } \\
(* *)\end{array}$} \\
\hline & & Sim & Não & & & & \\
\hline \multirow[t]{2}{*}{ Anti-hipertensivos $\left({ }^{*}\right)$} & Sim & 10 & 2 & & & & \\
\hline & Não & 2 & 220 & 98,3 & 0,82 & $0,61-0,96$ & 0,97 \\
\hline \multirow[t]{2}{*}{ Diuréticos } & Sim & 72 & 9 & & & & \\
\hline & Não & 23 & 130 & 86,3 & 0,71 & $0,61-0,79$ & 0,73 \\
\hline \multirow{4}{*}{$\begin{array}{l}\text { Beta-bloqueadores } \\
\text { adrenérgicos } \\
\text { Bloqueadores dos canais de } \\
\text { cálcio }\end{array}$} & Sim & 38 & 12 & & & & \\
\hline & Não & 6 & 178 & 92,3 & 0,76 & $0,65-0,85$ & 0,85 \\
\hline & Sim & 42 & 8 & & & & \\
\hline & Não & 9 & 175 & 92,7 & 0,79 & $0,68-0,88$ & 0,85 \\
\hline \multirow{4}{*}{$\begin{array}{l}\text { Inibidores da enzima } \\
\text { conversora da angiotensina } \\
\text { Hipolipemiantes }\end{array}$} & $\operatorname{Sim}$ & 71 & 8 & & & & \\
\hline & Não & 10 & 145 & 92,3 & 0,83 & $0,75-0,90$ & 0,85 \\
\hline & Sim & 18 & 5 & & & & \\
\hline & Não & 5 & 206 & 95,7 & 0,76 & $0,60-0,89$ & 0,91 \\
\hline \multirow[t]{2}{*}{ Hipoglicemiantes orais } & Sim & 27 & 1 & & & & \\
\hline & Não & 2 & 204 & 98,7 & 0,94 & $0,87-1,00$ & 0,97 \\
\hline \multirow[t]{2}{*}{ Insulinas } & Sim & 3 & 1 & & & & \\
\hline & Não & 0 & 230 & 99,6 & 0,86 & $0,73-0,98$ & 0,99 \\
\hline \multirow{2}{*}{$\begin{array}{l}\text { Antiinflamatórios não } \\
\text { esteróides }\end{array}$} & Sim & 25 & 24 & & & & \\
\hline & Não & 14 & 171 & 83,8 & 0,47 & $0,31-0,61$ & 0,68 \\
\hline
\end{tabular}

$\left(^{*}\right)$ metildopa, clonidina, rilmenidina, hidralazina e doxazosina. / (*) methyldopa, clonidine, rilmenidine, hydralazine and doxazosin.

$\left({ }^{*}\right)$ PABAK = kappa ajustado pelo efeito de prevalências e viés do observador $/\left({ }^{* *}\right)$ PABAK $=$ prevalence-adjusted and bias-adjusted kappa 
Tabela 5 - Valor preditivo positivo, sensibilidade e especificidade das informações contidas no questionário postal referentes ao uso de classes de medicamentos, por aposentados pelo Instituto Nacional do Seguro Social, com 60 anos de idade ou mais, Rio de Janeiro, RJ, Brasil, 2003.

Table 5 - Positive predictive value, sensitivity and specificity of the information contained in the postal questionnaire on drug class use by social security retirees at least 60 years old, Rio de Janeiro City, Brazil, 2003.

\begin{tabular}{|c|c|c|c|}
\hline Subgrupos terapêuticos & & Questionário post & \\
\hline & $\begin{array}{l}\operatorname{VPP}\left(\mathrm{N}^{*}\right) \\
\mathrm{IC}\end{array}$ & $\begin{array}{c}\text { Sensibilidade }\left(\mathrm{N}^{* *}\right) \\
\text { IC }\end{array}$ & $\begin{array}{l}\text { Especificidade }\left(\mathrm{N}^{* * *}\right) \\
\text { IC }\end{array}$ \\
\hline Anti-hipertensivos & $\begin{array}{c}0,83(12) \\
{[0,55-0,95]}\end{array}$ & $\begin{array}{c}0,83(12) \\
{[0,55-0,95]}\end{array}$ & $\begin{array}{c}0,99(222) \\
{[0,97-1,00]}\end{array}$ \\
\hline Diuréticos & $\begin{array}{c}0,89(81) \\
{[0,80-0,94]}\end{array}$ & $\begin{array}{c}0,76(95) \\
{[0,66-0,83]}\end{array}$ & $\begin{array}{c}0,94(139) \\
{[0,88-0,97]}\end{array}$ \\
\hline $\begin{array}{l}\text { Beta-bloqueadores } \\
\text { adrenérgicos }\end{array}$ & $\begin{array}{c}0,76(50) \\
{[0,63-0,86]}\end{array}$ & $\begin{array}{c}0,86(44) \\
{[0,73-0,94]}\end{array}$ & $\begin{array}{c}0,94(190) \\
{[0,89-0,96]}\end{array}$ \\
\hline $\begin{array}{l}\text { Bloqueadores seletivos } \\
\text { do canal de cálcio }\end{array}$ & $\begin{array}{c}0,84(50) \\
{[0,71-0,92]}\end{array}$ & $\begin{array}{c}0,82(51) \\
{[0,70-0,90]}\end{array}$ & $\begin{array}{c}0,96(183) \\
{[0,92-0,98]}\end{array}$ \\
\hline $\begin{array}{l}\text { Inibidores da enzima } \\
\text { conversora de } \\
\text { angiotensina }\end{array}$ & $\begin{array}{c}0,90(79) \\
{[0,81-0,95]}\end{array}$ & $\begin{array}{c}0,88(81) \\
{[0,79-0,93]}\end{array}$ & $\begin{array}{c}0,95(153) \\
{[0,90-0,97]}\end{array}$ \\
\hline Hipolipemiantes & $\begin{array}{c}0,78(23) \\
{[0,58-0,90]}\end{array}$ & $\begin{array}{c}0,78(23) \\
{[0,58-0,90]}\end{array}$ & $\begin{array}{c}0,98(211) \\
{[0,95-0,99]}\end{array}$ \\
\hline Hipoglicemiantes orais & $\begin{array}{c}0,96(28) \\
{[0,82-0,99]}\end{array}$ & $\begin{array}{c}0,93(29) \\
{[0,78-0,98]}\end{array}$ & $\begin{array}{c}1,00(205) \\
{[0,97-1,00]}\end{array}$ \\
\hline Insulinas & $\begin{array}{c}0,75(4) \\
{[0,30-0,95]}\end{array}$ & $\begin{array}{c}1,00(3) \\
{[0,44-1,00]}\end{array}$ & $\begin{array}{c}1,00(231) \\
{[0,98-1,00]}\end{array}$ \\
\hline Antiinflamatórios não esteróides & $\begin{array}{c}0,51(49) \\
{[0,37-0,64]}\end{array}$ & $\begin{array}{c}0,64(39) \\
{[0,48-0,77]}\end{array}$ & $\begin{array}{c}0,88(195) \\
{[0,82-0,92]}\end{array}$ \\
\hline
\end{tabular}

VPP $=$ Valor preditivo positivo $/ P P V=$ Positive predictive value

$\left(\mathrm{N}^{*}\right)$ número de indivíduos que acusaram o uso do medicamento no questionário postal. / ( $\left.N^{*}\right)$ number of individuals who reported drug use in the postal questionnaire.

$\left(\mathrm{N}^{* *}\right)$ número de indivíduos que acusaram o uso do medicamento na entrevista domiciliar. / ( $\left.\mathrm{N}^{* *}\right)$ number of individuals who reported drug use in the home interview.

$\left(\mathrm{N}^{* * *}\right)$ número de indivíduos que não acusaram o uso do medicamento na entrevista domiciliar. / $\left(N^{* * *}\right)$ number of individuals who did not report drug use in the home interview

IC = Intervalo de Confiança / Cl= Confidence Interval

Na Tabela 5 encontram-se os valores da sensibilidade, da especificidade e do valor preditivo positivo (VPP). A validade da abordagem postal foi elevada, chegando a ser perfeita no caso das insulinas. No caso dos anti-hipertensivos e dos hipoglicemiantes orais, a sensibilidade foi superior a $80 \%$ e a especificidade foi $99 \%$ e $100 \%$ respectivamente. Os menores índices de validade foram obtidos para o uso de antiinflamatórios não esteróides (VPP $=0,51 \%$; sensibilidade $=0,64 \%$; especificidade $=0,88 \%$ ).

\section{Discussão}

A taxa de resposta ao questionário postal foi $34,4 \%$. Estudos internacionais mostraram taxas mais elevadas $\left(44 \%^{7} ; 83,5 \%^{18}\right.$; e $\left.91,6 \%^{19}\right)$. Uma das razões que explica essa diferença se deve ao fato de que alguns países utilizam este meio para obter de forma rotineira informações inter-censitárias, como nos EUA e na Inglaterra, e conseqüentemente há maior taxa de resposta. Há que se considerar também que o nível de escola- 
ridade das populações desses países (Tabela 1) é elevado, o que também pode influenciar a taxa de resposta ${ }^{7}$. Embora a recusa já tenha sido apontada como sendo maior em inquéritos postais com população muito idosa, há estudos conduzidos em indivíduos com idade igual ou superior a setenta e cinco anos ${ }^{4,18}$ que obtiveram altas taxas de resposta ao questionário postal $(87,4 \%$; $\left.83,5 \%{ }^{18}\right)$. Asch ${ }^{20}$ observa que "a baixa taxa de resposta em inquéritos não significa uma representatividade obrigatoriamente baixa da amostra final e que, nesses casos, a representatividade da população de interesse pode ser até maior do que a obtida em estudos transversais com altas taxas de resposta". Esse autor concluiu que, sendo a taxa de resposta a melhor indicação indireta do viés de não-resposta, deve-se dar maior atenção à análise desse viés, detendo-se menos especificamente na taxa de resposta dos estudos. No presente estudo não houve diferenças significativas entre o grupo que respondeu ao inquérito postal (275) e o que não respondeu ao postal (290), segundo dados do cadastro da DATAPREV, quanto ao sexo, idade e valor do benefício (vide Métodos). A comparação entre todos os questionários postais preenchidos $(234+$ $41=275$ ) e os domiciliares preenchidos que não responderam ao postal $(290=524-234)$ é uma forma de analisar o viés de recusa ao inquérito postal. Assim, foi possível verificar que não houve diferença significativa entre esses dois grupos para as informações quanto às prevalências de uso das nove classes de fármacos analisadas neste estudo $(p=0,81)$, bem como para as prevalências de hipertensão arterial $(p=0,15)$ e diabetes mellitus (DM) $(\mathrm{p}=0,08)$ entre ambos os grupos. Além disso, as prevalências no grupo que só respondeu ao postal e no grupo que só respondeu ao domiciliar, para hipertensão arterial (67\% e $56 \%$ respectivamente) e DM (18\% e 15\% respectivamente) são semelhantes às encontradas entre os acima de 60 anos no município do Rio de Janeiro $(62,7 \%$ para hipertensão arterial ${ }^{21}$ e $16 \%$ para $\mathrm{DM}^{22}$ ) e no Brasil (65\% para hipertensão $\operatorname{arterial}^{23} \mathrm{e}$ $15,7 \%$ para $\left.\mathrm{DM}^{22}\right)$.
A extensão do questionário postal é apontada como causa de baixa taxa de resposta em inquéritos postais, especialmente com idosos ${ }^{4}$. No presente estudo, o postal era composto por 22 páginas, porém os caracteres eram maiores do que os habitualmente empregados, para permitir uma leitura confortável. Segundo alguns autores ${ }^{4,24}$, uma forma de elevar a taxa de resposta com questionários postais é a utilização de estratégias que estimulem a participação, como envio de cartões pelo correio e mensagens telefônicas aos retardatários. Apesar de estas iniciativas representarem um aumento de custos, os ganhos logísticos resultantes de sua aplicação devem ser considerados e podem justificar o seu emprego ${ }^{24}$.

Os achados apresentados permitem concluir que houve boa concordância e validade entre as informações sobre medicamentos, obtidas com os questionários postal e domiciliar. É provável que a solicitação para o entrevistado apresentar embalagens, recipientes ou bulas dos medicamentos usados na entrevista, ou para colocar diante de si embalagens e recipientes no postal, tenham contribuído para melhorar a validade do estudo.

A avaliação da concordância mostrou que a técnica postal é capaz de reproduzir dados com alta consistência para variáveis objetivas, como escolaridade e idade, e com boa consistência para informação sobre número de medicamentos. Estes resultados se assemelham aos de estudos que analisaram a informação postal dada por idosos sobre variáveis sociodemográficas $\left(k=0,93^{19} ; \mathrm{k}\right.$ $\left.=0,94^{4}\right)$ e número de medicamentos $(\mathrm{k}=$ $0,77^{19} ; \mathrm{k}=0,66^{4}$ ).

A confiabilidade das informações contidas no postal e na entrevista domiciliar referentes às classes de medicamentos analisadas mostrou-se elevada e consistente, com percentuais de concordância simples acima de $80 \%$, ultrapassando $90 \%$ em algumas classes. Outro estudo encontrou percentuais de concordância simples semelhantes para os anti-hipertensivos $(90 \%)^{25}$, para os hipoglicemiantes orais $(80 \%)^{25} \mathrm{e}$ para as insulinas $(100 \%)^{25}$. O índice 
kappa simples para os anti-hipertensivos $(0,82)$ foi superior ao encontrado em outro estudo $(0,60)^{26}$ conduzido com mulheres idosas aposentadas nos EUA, que comparou entrevista face a face com registro médico.

A sensibilidade do questionário postal para uso de hipoglicemiantes orais e de insulinas foi maior do que para anti-hipertensivos, resultado semelhante ao observado em um estudo norte-americano ${ }^{27}$. Isso talvez possa ser explicado pelo fato de que os portadores de DM seriam mais informados quanto aos possíveis danos causados por um tratamento irregular. Além disso, o tratamento do DM envolve menor troca de medicamentos quando comparado a outras doenças crônicas, como a hipertensão arterial. A sensibilidade para os antiinflamatórios não hormonais foi a menor encontrada entre as classes estudadas, resultado similar ao estudo de Johnson e Vollmer ${ }^{27}(67 \%)$, talvez em função do seu uso intermitente ou esporádico para tratamento de sintomas osteoarticulares, comuns entre pessoas acima de 60 anos de idade.

O número de respostas falso-negativas quanto ao uso de determinados medicamentos (bloqueadores dos canais de cálcio - 9; inibidores da enzima conversora de angiotensina - 10; diuréticos - 23) pode ser explicado pela dinâmica que envolve o seu manejo clínico e o tempo transcorrido entre as duas abordagens. As discordâncias podem ser devidas a mudanças no tratamento, surgimento de efeitos colaterais, ou dificuldades de aquisição.

A técnica considerada padrão-ouro para testar a validade das informações postais sobre o uso de medicamentos foi a entrevista face a face, embora a dosagem do nível sangüíneo do medicamento seja considerada mais válida ${ }^{28}$. Essa técnica, no entanto, é cara e demorada quando comparada a outras. O inventário realizado em entrevistas face a face tem sido considerado válido para a obtenção de informações sobre o uso de medicamentos ${ }^{1,25,27}$, sendo especialmente sensível para os anti-hipertensivos e os cardiovasculares quando comparado à dosagem do nível sérico das substâncias ${ }^{28}$.
Psaty e colaboradores ${ }^{1}$ compararam a informação sobre o uso de beta-bloqueadores adrenérgicos e de beta-agonistas (baseada apenas na capacidade de lembrar) com o inventário de medicamentos feito durante a entrevista domiciliar, usando como padrãoouro a freqüência cardíaca do usuário. Observaram que a informação sobre o uso destes fármacos obtida no inventário estava associada a um efeito significativo sobre a média da freqüência cardíaca $(\mathrm{p}<0,001)$. Além disso, Johnson e Vollmer ${ }^{27}$ compararam informações postais com registros farmacêuticos, utilizando o inventário de medicamentos na entrevista como padrãoouro. Concluíram que o postal foi mais apropriado que os registros.

Comparar a informação sobre o uso de medicamentos, tanto na descrição específica de cada classe quanto na comparação do número de medicamentos utilizados são formas distintas de examinar a questão da consistência dessa informação, e que traduzem na prática questões de enorme relevância como a possibilidade de descrição do perfil de uso, e a utilização irracional de medicamentos. No presente estudo os indivíduos informaram um número maior de medicamentos no postal do que na entrevista face a face, embora sem significância estatística, o que difere de outros estudos $^{19,27}$. Uma possível explicação é a crença, por parte do participante, de que a informação dada no postal estaria relacionada com algum tipo de distribuição de medicamentos, uma vez que o estudo foi patrocinado pelo Ministério da Saúde e pela FIOCRUZ, produtora e distribuidora de medicamentos. No questionário postal havia a orientação para o participante colocar à sua frente todas as embalagens ou as receitas de todos os medicamentos utilizados nos últimos quinze dias. Isso, porém, não impede a possibilidade da inclusão de outros fármacos que por ventura o participante tenha utilizado e lembrado no momento em que preenchia o questionário, momento esse que tanto pode ser de alguns minutos como de algumas horas. Essa possibilidade é mais remota na entrevista face a face, uma 
vez que o entrevistador registrava todos os medicamentos mediante as embalagens, bulas e receitas que o idoso dispunha no domicílio, num período de tempo limitado à presença do entrevistador.

Com o auxílio do método desenvolvido por Luiz e colaboradores ${ }^{15}$, concluiu-se que $80 \%$ das discordâncias entre as duas abordagens foram para diferenças de até três medicamentos. As discordâncias podem refletir o uso de fármacos em situações passageiras, como nas doenças agudas, ou naquelas de caráter recidivante, como é o caso das doenças reumáticas. O inquérito postal reproduziu bem a informação sobre os medicamentos de uso crônico, empregados no tratamento de doenças como DM e hipertensão arterial, porém o mesmo não ocorreu com os antiinflamatórios. Isso significa dizer que as discordâncias encontradas podem refletir "contaminação" das respostas por medicamentos de uso esporádico, ou sintomático. A diferença de dois meses entre o envio dos questionários pelo correio e a realização da entrevista domiciliar explica em parte a variabilidade nas respostas entre os dois métodos. As altas sensibilidade e especificidade do postal para as informações referentes aos medicamentos hipoglicemiantes orais e cardiovasculares parecem corroborar estas explicações. Assim, a aceitação de um limite para a diferença entre os dois métodos de até cinco medicamentos, por exemplo, pressupõe a inclusão de $90 \%$ de todas as divergências.

Tanto na abordagem postal quanto na domiciliar, observou-se um grande percentual de uso de cinco medicamentos ou mais. A utilização de cinco ou mais fármacos pode denotar uso inadequado, maior no postal (49\%) do que na entrevista (40\%). Um estudo norte americano de abrangência nacional ${ }^{29}$ apontou associação positiva entre o número de medicamentos usados e o número de medicamentos inapropriados, e concluiu que os fármacos para alívio da dor e aqueles dirigidos ao sistema nervoso central são os mais comumente usados sem justificativas técnicas.

Nossos resultados devem ser extrapolados com restrição para a população idosa geral do Estado ou do país, por se tratar de uma amostra composta por aposentados pelo sistema oficial de previdência social, residentes no município do Rio de Janeiro. Trata-se de uma população que recebe uma renda mensal fixa, o que pode, até certo ponto, gerar melhor qualidade na informação fornecida, e melhor cumprimento das prescrições e continuidade do tratamento, quando comparada à população geral.

Devido à escassez de estudos, inclusive em países desenvolvidos, nos quais o padrão-ouro é a entrevista face a face, torna-se difícil fazer outras comparações. Entretanto, observou-se que a via postal é uma abordagem confiável e válida. Embora no Brasil o uso do correio para coletar informações em pesquisas de saúde seja raro, espera-se que outros inquéritos sejam realizados e procedam à análise de concordância e validade, para que se possa, no futuro, testar sua utilidade de forma mais abrangente.

Agradecimentos: Os autores agradecem a participação de Carlos Henrique Klein no planejamento da pesquisa, de Andréia Queiroz Ribeiro e Célia Regina de Andrade na coleta de dados, e de Cristiano Moura de Soares no desenvolvimento do banco de dados.

\section{Referência}

1. Psaty BM, Lee M, Savage PJ, Rutan GM, German PS, Lyles M. Assessing the use of medications in the elderly: methods and initial experience in the cardiovascular health study. J Clin Epidemiol 1992; 45(6): 683-92.
2. Siemiatycki J, Campbell S. Nonresponse bias and early versus all responders in mail and telephone surveys. Am J Epidemiol 1984; 120(2): 291-301. 
3. Galán I, Rodriguez-Artalejo F, Zorilla B. Comparación entre encuestas telefónicas y encuestas "cara a cara" domiciliarias en la estimación de hábitos de salud e prácticas preventivas. Gac Sanit 2004; 18(6): 440-50.

4. Hébert R, Bravo G, Korner-Bitensky N, Voyer, L. Refusal and information bias associated with postal questionnaires and face-to-face interviews in very elderly subjects. J Clin Epidemiol 1996; 49(3): 373-81.

5. O'Toole BI, Battistutta D, Long A, Crouch K. A comparison of costs and data quality of three health survey methods: mail, telephone and personal home interview. Am J Epidemiol 1986; 124(2): 317-29.

6. Scott C. Research on mail survey. J R Statistic Society 1961; 124: 143-95.

7. Picavet HSJ. National Health surveys by mail or home interview: effects on response. J Epidemiol Community Health 2001; 55: 408-13.

8. Barber JH, Wallis JB, McKeating E. A postal screening questionnaire preventive geriatric care. J R Coll Gen Pract 1980; 30: 49-51.

9. Acurcio FA, Rozenfeld S, Ribeiro AQ, Klein CH, de Moura CS, de Andrade CR. Utilização de medicamentos por aposentados brasileiros. 1- Metodologia e resultados de cobertura de inquérito multicêntrico. Cad Saúde Pública 2006; 22(1): 87-96.

10. Bartko JJ, Carpenter WT. On the methods and theory of reliability. J Nerv Ment Dis 1976; 163(5): 307-17.

11. Byrt T, Bishop J, Carlin JB. Bias, prevalence and kappa. J Clin Epidemiol 1993; 46(5): 423-9.

12. Cohen J. Weighted kappa: nominal scale agreement with provision for scaled disagreement or partial credit. Psychol Bull 1968; 70: 213-20.

13. Byrt T. How good is agreement? Epidemiology 1996; 7 : 561.

14. Shrout PE, Fleiss JL. Intraclass Correlations: Uses in Assessing Rater Reliability. Psychol Bull 1979; 86(2): 4208.

15. Luiz RR, Costa AJL, Kale PL, Werneck GL. Assessment of agreement of a quantitative variable: a new graphical approach. J Clin Epidemiol 2003; 56: 963-7.

16. Luiz RR, Szklo M. More than one statistical strategy to assess agreement of quantitative measurements may usefully be reported. J Clin Epidemiol 2005; 58: 215-6.

17. Szklo M, Nieto FJ. Epidemiology: beyond the basics. Maryland: Aspen Publishers; 2000.
18. Smeeth L, Fletcher AE, Stirling S, Nunes M, Breeze E, Ng E, Bulpitt CJ, Jones D. Randomised comparison of three methods of administering a screening questionnaire to elderly people: findings from the MRC trial of the assessment and management of older people in the community. BMJ 2001; 323: 1403-7.

19. Doll H, McPherson K, Davies J, Flood A, Smith J, Williams G, et al. Reliability of questionnaire responses as compared with interview in the elderly: views of the outcome of transurethral resection of the prostate. Soc Sci Med 1991; 33(11): 1303-8.

20. Asch DA, Jedrziewski MK, Christakis NA. Response rates to mail surveys published in medical journals. J Clin Epidemiol 1997; 50: 1129-36.

21. Passos VMA, Assis TD, Barreto SM. Hipertensão arterial no Brasil: estimativa de prevalência a partir de estudos de base populacional. Epidemiol Serv Saúde 2006; 15(1): $35-45$.

22. Nucci LB, Toscano CM, Maia ALM, Fonseca CD, Britto MMB, Duncan BB, Schimidt MI. A nationwide population screening program for diabetes in Brazil. Rev Panam Salud Publica 2004; 16(5): 320-7

23. Serro Azul, J B; Paula, R S. Hipertensão arterial no idoso. In: Sociedade de Cardiologia do Estado de Säo Paulo. SOCESP: cardiologia. Rio de Janeiro: Atheneu; 1996

24. Siemiatycki J. A comparison of mail, telephone, and home interview - Strategies for household health surveys. Am J Public Health 1979; 69(3): 238-45.

25. Landry JA, Smyer MA, Tubman JG, Lago DJ, Roberts J, Simonson W. Validation of two methods of data collection of self-reported medicine use among the elderly. The Gerontologist 1988; 28(5): 672-6.

26. Paganini-Hill A, Ross RK. Reliability of recall of drug usage and other health-related information. Am J Epidemiol 1982; 116(1): 114-22.

27. Johnson RE, Vollmer WM. Comparing sources of drug data about the elderly. J Am Geriatric Soc 1991; 39: 107984.

28. Smith NL, Psaty BM, Heckbert SR, Tracy RP, Cornell ES. The reliability of medication inventory methods compared to serum levels of cardiovascular drugs in the elderly. J Clin Epidemiol 1999; 52(2); 143-6.

29. Goulding M. R. Inappropriate Medication Prescribing for Elderly Ambulatory Care Patients. Arch Intern Med 2004; 164: 305-12.

Recebido em: 02/03/09

Versão final reapresentada em: 23/07/09

Aprovado em: 30/07/09 